\title{
Temperonic Crystal: A Superlattice for Temperature Waves in Graphene
}

\author{
Marco Gandolfi®, ${ }^{1,2, *}$ Claudio Giannetti® ${ }^{3,4}$ and Francesco Banfi $\odot^{5, \dagger}$ \\ ${ }^{1}$ CNR-INO (National Institute of Optics), Via Branze 45, 25123 Brescia, Italy \\ ${ }^{2}$ Department of Information Engineering, University of Brescia, Via Branze 38, 25023 Brescia, Italy \\ ${ }^{3}$ Department of Physics, Università Cattolica del Sacro Cuore, Via Musei 41, 25121 Brescia, Italy \\ ${ }^{4}$ Interdisciplinary Laboratories for Advanced Materials Physics (I-LAMP), Università Cattolica del Sacro Cuore, \\ Via Musei 41, 25121 Brescia, Italy \\ ${ }^{5}$ FemtoNanoOptics group, Université de Lyon, CNRS, Université Claude Bernard Lyon 1, \\ Institut Lumière Matière, F-69622 Villeurbanne, France
}

(Received 31 August 2020; accepted 1 December 2020; published 31 December 2020)

\begin{abstract}
The temperonic crystal, a periodic structure with a unit cell made of two slabs sustaining temperature wavelike oscillations on short timescales, is introduced. The complex-valued dispersion relation for the temperature scalar field is investigated for the case of a localized temperature pulse. The dispersion discloses frequency gaps, tunable upon varying the slabs' thermal properties. Results are shown for the paradigmatic case of a graphene-based temperonic crystal. The temperonic crystal extends the concept of superlattices to the realm of temperature waves, allowing for coherent control of ultrafast temperature pulses in the hydrodynamic regime at above liquid nitrogen temperatures.
\end{abstract}

DOI: $10.1103 /$ PhysRevLett.125.265901

Coherent control of wavelike phenomena via metamaterials is driving, ever since four decades, a technological revolution in fields ranging from electronics, photonics, to phononics [1-6]. Although temperature has been historically considered as the paradigmatic example of an incoherent field, undergoing diffusive as opposed to wavelike propagation, on short space and timescales Fourier law fails [7-11] and the possibility for temperature wavelike propagation sets in [12-16]. The ultimate goal is to devise metamaterials, addressed as temperonic metamaterials, enabling coherent control of temperature oscillations arising in the hydrodynamic heat transport regime and operating at above liquid nitrogen temperature.

To this end the temperonic crystal (TC), a periodic structure based on a unit cell (u.c.) composed of two slabs sustaining temperature wavelike oscillations on short timescales, is here introduced as an archetypal example. The TC is the analog, for the temperature case, of electronic, phononic, and photonic superlattices. The complex-valued dispersion relation for the temperature scalar field in TCs is investigated for the case of a localized temperature pulse. The dispersion discloses frequency gaps, tunable upon varying the slabs thermal properties and dimensions, serving, for instance, as a frequency filter for a temperature pulse triggered by an ultrashort laser. Results are shown for

Published by the American Physical Society under the terms of the Creative Commons Attribution 4.0 International license. Further distribution of this work must maintain attribution to the author(s) and the published article's title, journal citation, and DOI. the realistic case of a graphene-based TC, graphene having recently shown to sustain temperature wavelike oscillations at above liquid nitrogen temperatures [15,17-20]. The TC serves as a conceptual building block to manipulate nanoscale heat transfer by exploiting interference effects.

In order to account for non-Fourier heat transport, occurring on short timescales and length scales, the present work relies on the dual-phase-lag (DPL) model [21-23]. The DPL introduces a causality relation between the onset of the heat flux, $\mathbf{q}$, and the temperature gradient, $\nabla T$ : $\mathbf{q}\left(\mathbf{x}, t+\tau_{q}\right)=-\kappa_{T} \nabla T\left(\mathbf{x}, t+\tau_{T}\right)$, where $\kappa_{T}$ is the thermal conductivity and $\tau_{T}$ and $\tau_{q}$ are positive build-up times for the inception of $\nabla T$ and $\mathbf{q}$, respectively [24], which microscopic attribution varies according to the specific physical system [25]. In the following, 1D propagation will be considered. Expanding the DPL model to first order $[26,27]$, while imposing local conservation of energy at time $t$, yields

$$
\left\{\begin{array}{l}
q+\tau_{q} \frac{\partial q}{\partial t} \simeq-\kappa_{T} \frac{\partial T}{\partial x}-\kappa_{T} \tau_{T} \frac{\partial^{2} T}{\partial x \partial t} \\
C \frac{\partial T}{\partial t}=-\frac{\partial q}{\partial x},
\end{array}\right.
$$

which may be recast as

$$
\left(\frac{\tau_{q}}{\alpha}\right) \frac{\partial^{2} T}{\partial t^{2}}-\frac{\partial^{2} T}{\partial x^{2}}+\frac{1}{\alpha} \frac{\partial T}{\partial t}-\tau_{T} \frac{\partial^{3} T}{\partial t \partial x^{2}}=0,
$$

with $C$ the volumetric specific heat and $\alpha=\kappa_{T} / C$ the thermal diffusivity. Whereas Eq. (2) is parabolic, its solution may still bear, under a practical standpoint, 


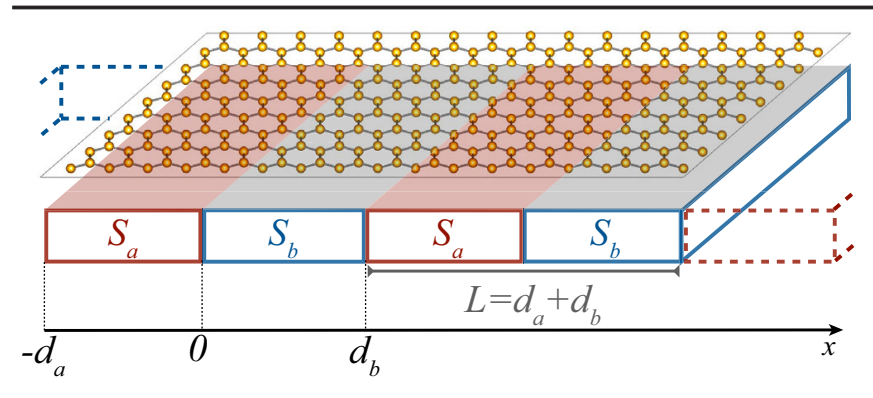

FIG. 1. Sketch of the temperonic crystal. Spatially periodic heating of a graphene layer leads to periodic stripes of length $d_{a}$, $d_{b}$, and temperature $T_{a}, T_{b}$, respectively, the u.c. dimension being $L=d_{a}+d_{b} . S_{j}=\left\{C, \kappa_{T}, \tau_{T}, \tau_{q}\right\}_{j}$ is the set of thermal properties of stripe $j$, with $j=\{\mathrm{a}, \mathrm{b}\}$. On a general basis, any periodic structure based on a unit cell composed of two slabs sustaining temperature wavelike oscillations on short timescales can serve as a TC.

wavelike characteristics $[28,29]$ provided $Z=\tau_{T} / \tau_{q}<$ $1 / 2$ [25]. This may be qualitatively understood recognising that the first two terms in Eq. (2) constitute the homogeneous wave equation, whereas the last two account for damping effects.

The wavelike propagation characteristics are better appreciated in reciprocal space. Seeking for solutions in the form $T(x, t)=\tilde{T}(\beta, \omega) \exp [i(\beta x+\omega t)]$ yields the dispersion relation for the homogeneous case

$$
\beta^{2}\left(1+i \omega \tau_{T}\right)=\left(\frac{\tau_{q}}{\alpha}\right) \omega^{2}\left(1-\frac{i}{\omega \tau_{q}}\right)
$$

linking the angular frequency $\omega$ and wave-vector $\beta$, both quantities being complex valued.

Based on the rationale that temperature propagation may preserve coherence properties in a homogenous slab, the concept of a TC is now introduced and its dispersion relation investigated in relation to the feasibility of opening frequencies band gaps in the temperature spectrum. The superlattice u.c., depicted in Fig. 1, is composed of two slabs $a$ and $b$, each now characterized by the set of thermal parameters $S_{j}=\left\{C, \kappa_{T}, \tau_{T}, \tau_{q}\right\}_{j}$ and length $d_{j}$, the subscript $j=\{\mathrm{a}, \mathrm{b}\}$ denoting the specific slab. The superlattice periodicity is $L=d_{a}+d_{b}$.

Within each slab, the thermal dynamics is described by the set of Eqs. (1) upon insertion of the slab's specific thermal parameters. Continuity of temperature and heat flux is enforced at the boundary of each slab. Furthermore, periodicity is accounted for applying Block-Floquet conditions, $T\left(x=d_{b}^{-}, t\right)=\exp (i k L) T\left(x=-d_{a}^{+}, t\right)$ and $q\left(x=d_{b}^{-}, t\right)=\exp (i k L) q\left(x=-d_{a}^{+}, t\right)$, leading to

$$
\begin{aligned}
& -\frac{1}{2}\left[\left(\frac{C_{a} \beta_{b}}{C_{b} \beta_{a}}\right)+\left(\frac{C_{a} \beta_{b}}{C_{b} \beta_{a}}\right)^{-1}\right] \sin \left(\beta_{b} d_{b}\right) \sin \left(\beta_{a} d_{a}\right) \\
& \quad+\cos \left(\beta_{b} d_{b}\right) \cos \left(\beta_{a} d_{a}\right)=\cos (k L),
\end{aligned}
$$

where $\beta_{j}$ is the wave vector in slab $j$. The $\beta_{j}$ are functions of $\omega$ via the dispersion relation for the homogeneous case, Eq. (3). Hence Eq. (4) is the dispersion relation in implicit form, linking the angular frequency $\omega$ and wave vector $k$ of the entire TC. For the sake of simplicity, the dispersion relation will be dealt in the frame of the extended scheme [30].

In this Letter, the interest is in the propagation of a temperature pulse $\delta T$ along the TC. The temperature pulse is assumed not to substantially affect the TC's temperature $T_{0}$; that is $\delta T \ll T_{0}$. A temperature pulse may be obtained as a linear superposition of plane waves. With reference to a single plane wave, the present scenario is properly accounted for assuming a real-valued $k$ and a complexvalued $\omega=\omega_{1}+i \omega_{2}$, where $2 \pi /\left|\omega_{1}\right|$ and $1 / \omega_{2}$ are the temperature's oscillation period and damping time, respectively [25]. The latter arises as a consequence of the diffusive and damping terms in Eq. (2) and sets the timescale over which wavelike behaviour can be observed. To quantify the damping of a given mode, the modal quality factor, $Q(k)=\left|\omega_{1}(k)\right| / \omega_{2}(k)$, is introduced. Numerical solution of Eq. (4) yields $\omega(k)$ and, ultimately, the dispersion curves $\omega_{1}(k)$ and $Q(k)$.

A qualitative understanding of the solution's relevant features may be achieved starting from the dispersion relation of a homogeneous effective material, obtained averaging the TC thermal parameters over the u.c. [31]: $\omega^{\mathrm{eff}}(k)=\omega_{1}^{\mathrm{eff}}(k)+i \omega_{2}^{\mathrm{eff}}(k), k$ being real valued. Both $\omega_{1}^{\mathrm{eff}}$ and the homogeneous effective material quality factor $Q^{\text {eff }}$ are continuous versus $k$ [25], i.e., there are no gaps. Upon adiabatically turning on the periodicity of the thermal parameters, $\omega^{\mathrm{eff}}(k)$ merges into $\omega(k)$ and frequency gaps in $\omega_{1}$, together with discontinuities in $Q$, open at the Brillouin zone (BZ) boundaries, that is at wave vectors $k_{n}^{\mathrm{BZ}}=\pi n / L$ with $n$ integer. The band-gap opening is meaningful provided the temperature wave is not overdamped: $Q\left(k_{n}^{\mathrm{BZ}}\right)$ [or $\left.Q^{\mathrm{eff}}\left(k_{n}^{\mathrm{BZ}}\right)\right]>1$ [32], the situation becoming similar to that of standard nondissipative superlattices [2,33]. A radically different behavior occurs for $k \ll k_{1}^{\mathrm{BZ}}$, that is for temperature's wavelengths $\lambda(=2 \pi / k) \gg L$. In this case interference effects are negligible and the wave "feels" the underlying homogeneous effective material. The TC dispersion is then expected to match that of the homogeneous effective material itself.

We now present a realistic design of a graphene-based $\mathrm{TC}$, where the previous concepts find application for the case of phononic temperatures waves [34]. Phonons temperature waves were recently reported in graphite at $80 \mathrm{~K}$ on the ultrafast timescale in the seminal work of Huberman et al. [15] and are expected to be sustained also in graphene [35-37]. The experimental evidences were successfully rationalized by Eq. (3) (with $\omega$ complex and $k$ real), upon identification of $\tau_{T}$ with $\tau_{N}$ and $\tau_{q}$ with $\tau_{U}$, where $\tau_{N}$ and $\tau_{Q}$ are the average phonon scattering times for normal $(N)$ and umklapp $(U)$ processes, respectively [25]. Both scattering 
TABLE I. Thermal parameters for graphene as a function of temperature. The temperatures have been chosen so as to fulfill the requirements for wavelike propagation: $Z<1 / 2$.

\begin{tabular}{lcccc}
\hline \hline Thermal parameter & $T=80 \mathrm{~K}$ & $T=100 \mathrm{~K}$ & $T=110 \mathrm{~K}$ & $T=120 \mathrm{~K}$ \\
\hline$\kappa_{T}[(\mathrm{~W} / \mathrm{m} \mathrm{K})][38]$ & $4.3 \times 10^{3}$ & $5.0 \times 10^{3}$ & $5.0 \times 10^{3}$ & $5.0 \times 10^{3}$ \\
$C\left[\left(\mathrm{~J} / \mathrm{m}^{3} \mathrm{~K}\right)\right][39]$ & $2.3 \times 10^{5}$ & $3.3 \times 10^{5}$ & $3.7 \times 10^{5}$ & $4.4 \times 10^{5}$ \\
$\tau_{T}[\mathrm{ps}][20,25]$ & 3.0 & 2.5 & 2.2 & 2.1 \\
$\tau_{q}[\mathrm{ps}][20,25]$ & 1800 & 300 & 140 & 90 \\
\hline \hline
\end{tabular}

times are temperature dependent. Onset of the wavelike regime requires $Z<1 / 2$, a condition met at cryogenic temperatures where $\tau_{U} \gg \tau_{N}$. Graphene can thus serve as a suitable building block for a TC operating at temperatures up to $120 \mathrm{~K}$, see Table I, and possibly beyond.

The superlattice is then obtained imposing a static, 1D space-periodic base temperature profile, $T_{0}(x)$, on a graphene layer, with $T_{0}(x)$ in the form of a square wave of period $L$, taking the values $T_{a}$ and $T_{b}$ over the extents $d_{a}$ and $d_{b}$, respectively [40]. Both $T_{a}$ and $T_{b}$ are chosen so as to ensure that $N$-scattering events prevail over $U$ ones (hence $Z \ll 1$ ). $T_{0}(x)$ leads to a periodic modulation of the graphene's sheet thermal parameters through their temperature dependence, ultimately resulting in the TC depicted in Fig. 1. Practically, $T_{0}(x)$ can be applied via a spaceperiodic temperature modulation of a supporting chip on which the graphene layer adheres. This can be achieved in a variety of ways: for instance, by exploiting the photothermal effect and shining an optical grating on a homogeneous substrate material kept at cryogenic temperature. Another possibility is by homogeneous illumination of a cryogenic substrate made of stripes with different absorption coefficients or via nanopatterning a periodic resistor array on the supporting substrate.

A specific case is now addressed, in which the u.c. slabs $a$ and $b$ are kept at temperature $T_{a}=100$ and $T_{b}=80 \mathrm{~K}$, respectively. For sake of simplicity, in what follows a symmetric u.c. is assumed: $d_{a}=d_{b}=L / 2$. Furthermore, graphite's thermal properties are taken (see Table I) as a good compromise for the wide range of graphene's thermal properties reported in the literature, the latter strongly depending on a plethora of parameters ranging from degree of crystallinity, production method, grain size to the kind of supporting material [41-44]. The values for $\tau_{T}$ and $\tau_{q}$ have been adopted [25] upon fitting the experimental dispersion relation [15] with Eq. (3), i.e., within the frame of the DPL. These values are consistent with the scattering times obtained in the frame of the Boltzmann transport equation [20]. The focus will be limited to opening of the first band gap, $n=1$, the discussion being similar for higher gaps.

The black dashed line in Fig. 2 represents $\omega_{1}^{\text {eff }}$ [panel (a)] and $Q^{\text {eff }}$ [panel (b)] vs $k$ for the homogeneous effective material. The u.c. size $L$ is chosen to be $5 \mu \mathrm{m}$, similarly to the transient grating periodicity exploited in Ref. [15] to observe temperature waves in graphite at $80 \mathrm{~K}$. The black full lines in Fig. 2 represent $\omega_{1}$ [panel (a)] and $Q$ [panel (b)] vs $k$ for the TC. In particular, a gap opens in the $\omega_{1}$ spectrum, implying that temperature waves with $\omega_{1}$ in the 1.8 to $3.5 \mathrm{Grad} / \mathrm{s}$ range are not supported by the TC. The gap extent $\Delta_{g}$ amounts to $1.7 \mathrm{Grad} / \mathrm{s}$ and is centered at $\sim \omega_{1}^{\text {eff }}\left(k_{1}^{\mathrm{BZ}}\right) \sim 2.5 \mathrm{Grad} / \mathrm{s}$, the latter being the value of $\omega_{1}^{\text {eff }}$ at the 1st BZ boundary. The periodicity is very effective in opening the band gap, the relevant figure of merit (FOM) being $\Delta_{g} /\left|\omega_{1}^{\text {eff }}\left(k_{1}^{\mathrm{BZ}}\right)\right| \sim 64 \%$. The TC hence works as an effective frequency filter for temperature waves operating on a timescale $\sim Q^{\text {eff }}\left(k_{1}^{\mathrm{BZ}}\right) /\left|\omega_{1}\left(k_{1}^{\mathrm{BZ}}\right)\right| \sim 2$ ns.
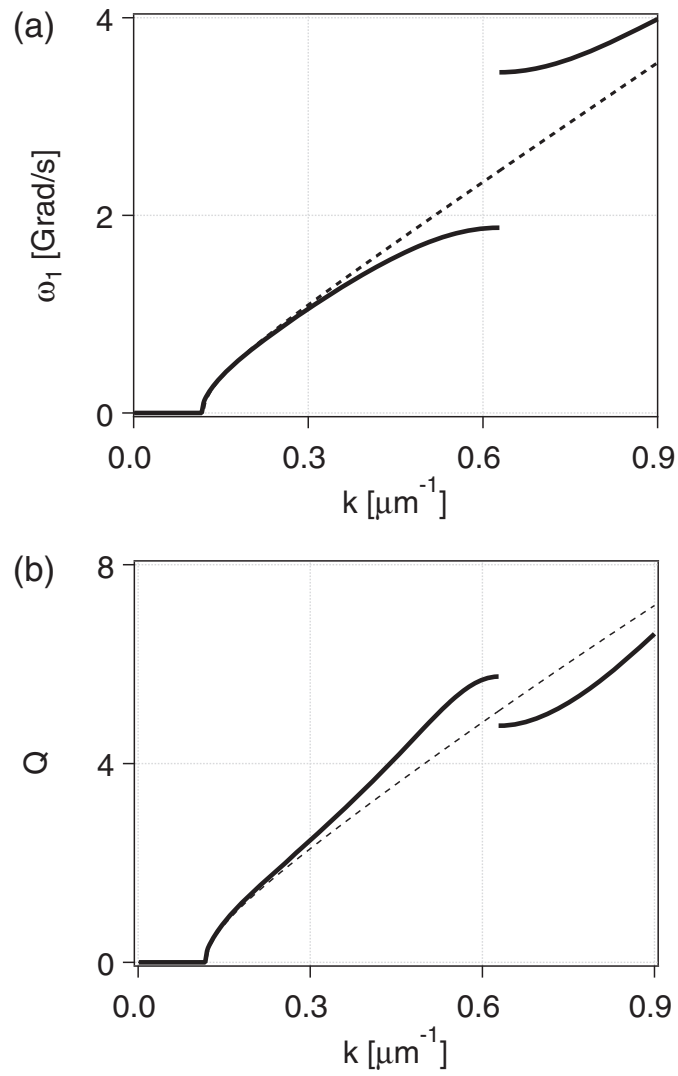

FIG. 2. Temperature band structure: dispersion relation $\omega_{1}$ (a) and quality factor $\mathrm{Q}$ (b). Black full line: graphene TC with $T_{a}=100$ and $T_{b}=80 \mathrm{~K}$. Black dashed line: $\omega_{1}^{\text {eff }}$ (a) and $Q^{\text {eff }}$ (b) for the corresponding homogeneous effective material. The unit cell size is $5 \mu \mathrm{m}$. 
For temperature wavelengths $\lambda \gg L\left(k \ll 2 k_{1}^{\mathrm{BZ}}\right)$ the TC dispersion relation matches that of the homogeneous effective material, as appreciated inspecting Fig. 2 for $k \lesssim 0.3 \mu \mathrm{m}^{-1}$. Within this regime one can thus engineer an artificial material sustaining temperature waves on a timescale $\sim Q^{\text {eff }}(k) /\left|\omega_{1}(k)\right|$, which effective thermal properties are obtained as an average over the u.c.

We now investigate the gap tunability in terms of spectral position and extent, upon varying the slabs' thermal properties via their temperature dependence. To this end, and following the same rationale as for the previous case, we report results for two additional TCs obtained keeping $T_{a}=100 \mathrm{~K}$ while augmenting $T_{b}$ to 110 and $120 \mathrm{~K}$, respectively. The u.c. size is chosen to be $L=2.1$ and $L=2 \mu \mathrm{m}$, respectively. On a general basis, augmenting the working temperature diminishes the phonon scattering times, hence allowing for a shorter $L$, provided the requirement $Z<1 / 2$ still holds. These periodicities are readily implementable with current nanopatterning techniques.

Figure 3 shows the normalized dispersion relations for all three TCs. For sake of comparison, $k$ and $\omega_{1}$ have been normalized against $k_{1}^{\mathrm{BZ}}$ and $\omega_{1}^{\mathrm{eff}}\left(k_{1}^{\mathrm{BZ}}\right)$, the latter two values being specific to each TC. The normalized dispersion relations of the three homogeneous effective materials are thus the same (black dashed line). The normalized band gaps correspond to the FOMs. As the temperature difference $\left|T_{b}-T_{a}\right|$ augments for a fixed value of $T_{a}$, so does the FOM, which is $27 \%, 46 \%$, and $64 \%$ for values of $T_{b}=110$ (red line), 120 (blue line), and $80 \mathrm{~K}$ (black line), respectively. Mind though that the FOM does not depend on the temperature difference only, but also on the actual slab temperatures. For instance, $\left|T_{b}-T_{a}\right|=20 \mathrm{~K}$ for the TCs with $T_{b}=80$ (full black line) and $120 \mathrm{~K}$ (full blue line) but the FOMs are different. The actual

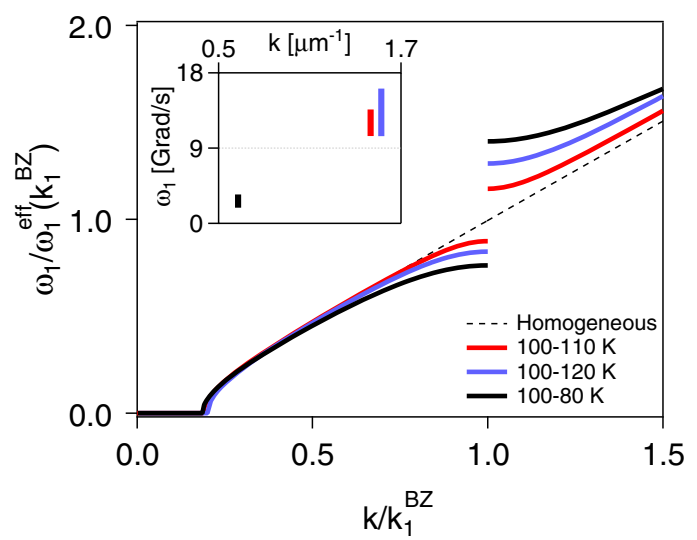

FIG. 3. Normalized dispersion relations for a graphene TC with $T_{a}=100 \mathrm{~K}$ kept fixed and $T_{b}=110$ (red full line), 120 (blue full line), and $80 \mathrm{~K}$ (black full line). Black dashed line: normalized dispersion relation for the corresponding homogeneous effective materials (same curve for all three cases). Inset: 1st gap location and extent (vertical bars) for each TC in actual, i.e., non-normalized, units. (i.e., non-normalized) band gaps are shown as vertical bars in the inset of Fig. 3. What emerges is that the band gap can be tuned, both in spectral position and extent, over a wide range of angular frequencies, all within reach of state-of-the art optothermal spectroscopies [14,15,17].

The temperonic crystal, a superlattice for temperature wavelike motion occurring in the hydrodynamic regime, was introduced. The band structure for temperature waves may be engineered tuning the TC thermal and geometrical properties. The concept was illustrated for the case of a graphene-based TC, where temperature frequency gaps in the $1-10 \mathrm{Grad} / \mathrm{sec}$ range and $Q$ factor $\sim 5$ were obtained for TC periodicities $\sim 5 \mu \mathrm{m}$ and working temperatures above that of liquid nitrogen. Tuning of the temperature band structure was demonstrated under realistic conditions.

The TC concept can be readily expanded to encompass other materials sustaining temperature waves in the hydrodynamic regime such as graphite and single-layer transition metal dichalcogenides [45], for the case of phononic temperature, and quantum materials [17,25], for the case of electronic and spin temperatures. For the latter two cases (quantum materials) the scattering times can be faster than the ones presently addressed, potentially allowing for a shorter TC periodicity, enhanced $Q$ factors, and requiring observation on faster timescales. TCs based on two-dimensional materials are particularly amenable for integration on nanostructured supports [46]. In this context twistedgraphene superlattices $[47,48]$ merge the properties of graphene and correlated materials offering the possibility of controlling the ratio between the local interaction strength and the effective bandwidth. This could provide enhanced thermal parameter tunability upon acting on the magic angle of twisted bilayer graphene.

The TC may be achieved also with alternate layout designs. For instance, one could envision creating the TC alternating two materials with a good contrast in their mutual thermal properties instead of imposing the periodicity via a thermal modulation of the same material.

In perspective, manipulation of temperature pulses on short time and length scales, by exploiting interference effects as the one here shown, opens up to thermal nanodevice concepts based on coherence effects such as waveguides, cavities, and frequency filters for the temperature field.

M. G. acknowledges financial support from the CNR Joint Laboratories program 2019-2021. C. G. acknowledges support from Università Cattolica del Sacro Cuore through D.2.2 and D.3.1 grants, from MIUR through PRIN 2015 (Prot 2015C5SEJJ001) and PRIN 2017 (Prot. 20172H2SC4_005) programs. Università Cattolica del Sacro Cuore contributed to the funding for open-access publication of this work. F. B. acknowledges financial support from Universite de Lyon in the frame of the IDEXLYON Project (ANR-16-IDEX-0005) and from Université Claude Bernard Lyon 1 through the BQR Accueil EC 2019 grant. 
*marco.gandolfi@ino.cnr.it

†rancesco.banfi@univ-lyon1.fr

[1] E. Istrate and E. H. Sargent, Rev. Mod. Phys. 78, 455 (2006).

[2] C. Weisbuch and B. Vinter, Quantum Semiconductor Structures: Fundamentals and Applications (Elsevier, New York, 2014).

[3] M. Maldovan, Nature (London) 503, 209 (2013).

[4] M. Wegener, Science 342, 939 (2013).

[5] N. I. Zheludev and Y. S. Kivshar, Nat. Mater. 11, 917 (2012).

[6] O. Matsuda, M. C. Larciprete, R. L. Voti, and O. B. Wright, Ultrasonics 56, 3 (2015).

[7] A. J. Minnich, J. A. Johnson, A. J. Schmidt, K. Esfarjani, M. S. Dresselhaus, K. A. Nelson, and G. Chen, Phys. Rev. Lett. 107, 095901 (2011).

[8] J. A. Johnson, A. A. Maznev, J. Cuffe, J. K. Eliason, A. J. Minnich, T. Kehoe, C. M. Sotomayor Torres, G. Chen, and K. A. Nelson, Phys. Rev. Lett. 110, 025901 (2013).

[9] K. M. Hoogeboom-Pot, J. N. Hernandez-Charpak, X. Gu, T. D. Frazer, E. H. Anderson, W. Chao, R. W. Falcone, R. Yang, M. M. Murnane, H. C. Kapteyn et al., Proc. Natl. Acad. Sci. U.S.A. 112, 4846 (2015).

[10] X. Chen, C. Hua, H. Zhang, N. K. Ravichandran, and A. J. Minnich, Phys. Rev. Applied 10, 054068 (2018).

[11] T. D. Frazer, J. L. Knobloch, K. M. Hoogeboom-Pot, D. Nardi, W. Chao, R. W. Falcone, M. M. Murnane, H. C. Kapteyn, and J. N. Hernandez-Charpak, Phys. Rev. Applied 11, 024042 (2019).

[12] Y. Zhang, X. Shi, W. You, Z. Tao, Y. Zhong, F. C. Kabeer, P. Maldonado, P. M. Oppeneer, M. Bauer, K. Rossnagel et al., Proc. Natl. Acad. Sci. U.S.A. 117, 8788 (2020).

[13] C. Hua and L. Lindsay, Phys. Rev. B 102, 104310 (2020).

[14] A. Beardo, M. López-Suárez, L. A. Pérez, L. Sendra, M. I. Alonso, C. Melis, J. Bafaluy, J. Camacho, L. Colombo, R. Rurali et al., arXiv:2007.05487.

[15] S. Huberman, R. A. Duncan, K. Chen, B. Song, V. Chiloyan, Z. Ding, A. A. Maznev, G. Chen, and K. A. Nelson, Science 364, 375 (2019).

[16] A. Beardo, M. G. Hennessy, L. Sendra, J. Camacho, T. G. Myers, J. Bafaluy, and F.X. Alvarez, Phys. Rev. B 101, 075303 (2020).

[17] M. Gandolfi, G. L. Celardo, F. Borgonovi, G. Ferrini, A. Avella, F. Banfi, and C. Giannetti, Phys. Scr. 92, 034004 (2017).

[18] S. Lee, D. Broido, K. Esfarjani, and G. Chen, Nat. Commun. 6, 6290 (2015).

[19] Z. Ding, J. Zhou, B. Song, V. Chiloyan, M. Li, T.-H. Liu, and G. Chen, Nano Lett. 18, 638 (2018).

[20] A. Cepellotti, G. Fugallo, L. Paulatto, M. Lazzeri, F. Mauri, and N. Marzari, Nat. Commun. 6, 6400 (2015).

[21] D. Y. Tzou, J. Heat Transfer 117, 8 (1995).

[22] D. Y. Tzou, J. Thermophys. Heat Transfer 9, 686 (1995).

[23] D. Y. Tzou, Macro-to Microscale Heat Transfer: The Lagging Behavior (John Wiley \& Sons, New York, 2014).

[24] J. Ordóñez-Miranda and J. J. Alvarado-Gil, Mech. Res. Commun. 37, 276 (2010).

[25] M. Gandolfi, G. Benetti, C. Glorieux, C. Giannetti, and F. Banfi, Int. J. Heat Mass Transfer 143, 118553 (2019).

[26] S. L. Sobolev, Phys. Usp. 40, 1043 (1997).

[27] M. A. Al-Nimr, M. Naji, and V. S. Arbaci, J. Heat Transfer 122, 217 (2000).
[28] D. Tang and N. Araki, Int. J. Heat Mass Transfer 42, 855 (1999).

[29] J. Ordóñez-Miranda and J. Alvarado-Gil, Int. J. Thermal Sci. 48, 2053 (2009).

[30] N. W. Ashcroft and N. D. Mermin, Solid State Phys. (Holt, Rinehart and Winston, New York, 1976).

[31] The homogeneous effective material is a homogeneous material whom thermal parameters are obtained as an average over the u.c. In other words, the generic thermal parameter of the homogeneous effective material $X^{\text {eff }}$ is obtained as $X^{\text {eff }}=\left(d_{a} X_{a}+d_{b} X_{b}\right) / L$, with $X_{j}$ the corresponding thermal parameter of slab $j$.

[32] Formally, the criteria $Q^{\text {eff }}\left(k_{1}^{\mathrm{BZ}}\right)=\max \left\{Q^{\text {eff }}(k)\right\}$ establishes the TC's periodicity enabling optimal band-gap opening at the 1st BZ boundary. Nevertheless, the so obtained $L$ may be too short to grant local thermalization, a premise for the DPL model to hold, and/or incompatible with technological constraints.

[33] S. Tamura, D. C. Hurley, and J. P. Wolfe, Phys. Rev. B 38, 1427 (1988).

[34] S. Volz, J. Ordonez-Miranda, A. Shchepetov, M. Prunnila, J. Ahopelto, T. Pezeril, G. Vaudel, V. Gusev, P. Ruello, E. M. Weig et al., Eur. Phys. J. B 89, 15 (2016).

[35] M. Simoncelli, N. Marzari, and A. Cepellotti, Phys. Rev. X 10, 011019 (2020).

[36] A. Cepellotti and N. Marzari, Phys. Rev. Mater. 1, 045406 (2017).

[37] A. Cepellotti and N. Marzari, Phys. Rev. X 6, 041013 (2016).

[38] G. Fugallo, A. Cepellotti, L. Paulatto, M. Lazzeri, N. Marzari, and F. Mauri, Nano Lett. 14, 6109 (2014).

[39] W. DeSorbo and W. Tyler, J. Chem. Phys. 21, 1660 (1953).

[40] A square wave variation of $T_{0}(x)$ allows a solution in analytical form, thus having the merit of highlighting the relevant physics. In a real case scenario, the spatial temperature modulation would though be smoother. This may possibly lead to a reduction of the band gaps frequency extent occurring between high order frequency bands, their frequency position remaining primarily determined by the TC periodicity.

[41] D. Nika, S. Ghosh, E. Pokatilov, and A. Balandin, Appl. Phys. Lett. 94, 203103 (2009).

[42] D. Kolesnikov and V. Osipov, Europhys. Lett. 100, 26004 (2012).

[43] J. H. Seol, I. Jo, A. L. Moore, L. Lindsay, Z. H. Aitken, M. T. Pettes, X. Li, Z. Yao, R. Huang, D. Broido et al., Science 328, 213 (2010).

[44] E. Pop, V. Varshney, and A. K. Roy, MRS Bull. 37, 1273 (2012).

[45] P. Torres, F. X. Alvarez, X. Cartoixà, and R. Rurali, 2D Mater. 6, 035002 (2019).

[46] X. Gu and R. Yang, Annual review of heat transfer 19, 1 (2016).

[47] Y. Cao, V. Fatemi, S. Fang, K. Watanabe, T. Taniguchi, E. Kaxiras, and P. Jarillo-Herrero, Nature (London) 556, 43 (2018).

[48] Y. Cao, V. Fatemi, A. Demir, S. Fang, S. L. Tomarken, J. Y. Luo, J. D. Sanchez-Yamagishi, K. Watanabe, T. Taniguchi, E. Kaxiras et al., Nature (London) 556, 80 (2018). 